\title{
A near-infrared fluorescent probe for evaluating glutamyl transpeptidase fluctuation in idiopathic pulmonary fibrosis cell and mice models
}

\author{
$\mathrm{Na} \mathrm{He}^{\mathrm{a}, \mathrm{d}, 1}$, Yude Wang ${ }^{\mathrm{a}, \mathrm{d}, 1}$, Yan Huang ${ }^{\mathrm{c}, * * *}$, Xiaoyan Wang ${ }^{\mathrm{c}}$, Lingxin Chen ${ }^{\mathrm{b}, \mathrm{c}, *}$, Changjun $\mathrm{Lv}^{\mathrm{a}, \mathrm{d}, * *}$ \\ ${ }^{a}$ Department of Respiratory Medicine, Binzhou Medical University Hospital, Binzhou, 256603, China \\ ${ }^{\mathrm{b}}$ CAS Key Laboratory of Coastal Environmental Processes and Ecological Remediation, Yantai Institute of Coastal Zone Research (YIC), Chinese Academy of Sciences \\ (CAS), Shandong Key Laboratory of Coastal Environmental Processes, YICCAS, Yantai, 264003, China \\ ${ }^{\mathrm{c}}$ School of Pharmacy, Binzhou Medical University, Yantai, 264003, China \\ ${ }^{\mathrm{d}}$ Medicine Research Center, Institute of Molecular Medicine, Binzhou Medical University, Yantai, 264003, China
}

A R T I C L E I N F O

\section{Keywords:}

Fluorescent probe

Near-infrared

Glutamyl transpeptidase

Idiopathic pulmonary fibrosis

Fluorescence imaging

\begin{abstract}
A B S T R A C T
Idiopathic pulmonary fibrosis (IPF), whose early diagnosis and effective treatment still remain the focus of clinical studies, is a chronic, irreversible and finally fatal pulmonary disease. Glutamyl transpeptidase (GGT) has a potential relationship with the occurrence and development of IPF. Therefore, explore whether GGT can be applied as a biological indicator for the clinical identification and diagnosis of IPF, sensitive and accurate detection of GGT under physiological conditions is necessary. In this research, a new fluorescent probe Cy-GGT was exhibited for detecting GGT concentrations in pulmonary fibrosis cell and mice models. Cy-GGT was capable of rapidly and selectively detecting GGT in vitro. The probe was successfully applied for visualizing GGT in oxidative stress cell models, pulmonary fibrosis cells and mice models. The results revealed that intracellular GGT increased in the cells and mice models of pulmonary fibrosis. GGT plays a significant part in pulmonary fibrosis, and the GGT level abnormally expressed in the lung tissue may be employed as a potential biological indicator to diagnose IPF lesions. Furthermore, the discovery of the close relationship between IPF and GGT will provide a new idea for effective therapy of IPF in the future.
\end{abstract}

\section{Introduction}

Idiopathic pulmonary fibrosis (IPF), acknowledged as a chronic, non-reversible, progressive, and ultimately lethal disease, is a most common type of the idiopathic interstitial pneumonia [1]. The median survival time of IPF is between 3-4 years from diagnosis and it largely occurs in older male adults. The incidence increases with age, from 1.1 new cases per 100,000 person-years in adults ages 18-34 to 19.3 new cases per 100,000 person-years in adults ages 55-64 [1]. Early diagnosis of IPF is vital to enable patients to get treatments that slow disease progression. However, since no single clinical feature is characteristic to IPF, the diagnosis needs a combination of chest imaging, clinical, and pathologic findings, which bring great difficulties to early and precise diagnosis [2]. In addition, current treatment options for IPF only slow down the progression of disease, alleviate suffering, avoid deterioration, and eventually extend survival $[3,4]$. Consequently, there is an urgent necessity to establish an accurate diagnosis and more effective therapies for IPF [5]. Although the cause of IPF is not entirely clear yet, the IPF patients have higher levels of oxidative stress than healthy people [6]. There is evidence that the pathogenesis of IPF is related to oxidative stress, and ROS generation by Nox4 mediates the activation of myofibroblasts and the fibrogenic responses to pulmonary injury [7]. It showed that the cellular oxidative stress state is important in the development of IPF.

Glutamyl transpeptidase (GGT), a membrane-relevant enzyme, plays a crucial role in a large number of pathological and physiological processes [8-11], involving regulating the metabolism of reduction and oxidation, diabetes, cancers and drug resistance [12-14]. Notably, the pathological state of oxidative stress could cause the increase of GGT level. GGT can maintain the metabolism and homeostasis of glutathione (GSH) in the cell, and thus can regulate the oxidative stress generated under pathological conditions. GSH is an endogenous antioxidant in

\footnotetext{
* Corresponding author at: CAS Key Laboratory of Coastal Environmental Processes and Ecological Remediation, Yantai Institute of Coastal Zone Research (YIC), Chinese Academy of Sciences(CAS), Shandong Key Laboratory of Coastal Environmental Processes, YICCAS, Yantai, 264003, China.

** Corresponding author at: Department of Respiratory Medicine, Binzhou Medical University Hospital, Binzhou, 256603, China.

*** Corresponding author.

E-mail addresses: huangyanpy@163.com (Y. Huang), 1xchen@yic.ac.cn (L. Chen), lucky_lcj@sina.com (C. Lv).

${ }^{1}$ These authors contributed equally.
} 
cells, which protects cells against oxidative stress by neutralizing the ROS and sustaining the exogenous antioxidant levels like vitamins $\mathrm{C}$ and E [15]. GGT sustains intracellular GSH levels by taking part in gamma-glutamyl cycle [16-18]. GGT, as an essential part of endogenous antioxidant system, is vital to understand main physiological resistance mechanism to oxidative stress damage in regulating a number of disease cycles. In addition, recent researches have suggested that bleomycin-induced pulmonary fibrosis is attenuated in GGT-deficient mice [19]. Therefore, we aim to explore the relationship between GGT and IPF.

Compared with the traditional detection method of GGT involving colorimetric assays (p-Nitroanilide-based) [20], high-performance liquid chromatography (HPLC) [21], and electrochemistry [22], fluorescence-based technology has received a great deal of attention because of its essential attributes of real-time imaging, rapid response, high selectivity, and non-damaging detection for biological target in complex samples [23-33]. Owing to their universal chemical structure modification, fluorescent probes are more likely to capture diverse biological targets [34-38]. This makes it possible to explore new design methods for detecting specific enzymes with fluorescent probes. Recently, the fluorescent probes have been demonstrated as efficient ways to tract enzymes in biological samples $[39,40]$. Up to now, many fluorescent probes have been designed to monitor GGT level by means of the characteristic cleavage of g-glutamate bond with a quenched fluorophore [41-49]. However, the vast majority of them were designed by means of fluorophores with short excitation wavelengths, which are likely to suffer from auto-fluorescent background, photo damage and attenuated signals. There are still some difficulties in accurately detecting GGT level in clinical and biological samples. To reduce these problems, the fluorescent probes with near-infrared (NIR) excitation and emission were developed to monitor intracellular GGT level in physiological environment [50-53]. According to our knowledge, although the emission wave of some published research located in NIR region, emission wavelength of NIR fluorescent probe exceed $750 \mathrm{~nm}$ were still lacking [54]. Therefore, there is an urgent need to synthesize a NIR fluorescent probe to selectively and accurately monitor intracellular GGT level in clinical and biological samples.

Hence, we conceived a NIR fluorescent probe Cy-GGT with $780 \mathrm{~nm}$ emission for detecting the behavior of GGT in cells and in vivo. By tracking GGT level in living cells and in vivo, the versatile function of Cy-GGT in organismal systems was detailedly assessed. Furthermore, Cy-GGT was applied to clarify the increase of GGT level in pulmonary fibrosis cell and mice models, revealing the close connection between GGT and IPF. To our knowledge, this is the first time to use a fluorescence probe for imaging and detecting the level of GGT in pulmonary fibrosis cell and mice models. More importantly, we provide clear and intuitive evidence for changes of GGT concentration in the development of pulmonary fibrosis, which may offer a new strategy for the specific diagnosis of IPF in clinical.

\section{Experimental methods}

\subsection{Synthesis of probe Cy-GGT}

We added compound e ( $87 \mathrm{mg}, 0.1 \mathrm{mM})$ to dry $\mathrm{CH}_{2} \mathrm{Cl}_{2}(2 \mathrm{~mL})$, then slowly stirred with a normal temperature for $10 \mathrm{~min}$ to ensure that compound e was completely dissolved. Furthermore, $\mathrm{CH}_{2} \mathrm{Cl}_{2}$ /TFA $(2 \mathrm{~mL}, \mathrm{v} / \mathrm{v}=1: 1)$ was dropped into the above mixtures. After the above operation was completed, this mixture was further continuously stirred overnight. To obtain the deep green solid probe Cy-GGT, we purified the preliminary product by silica gel column chromatography. (64 mg, $90 \%$ yield). ${ }^{1} \mathrm{H}$ NMR $\left(\mathrm{CDCl}_{3}-\mathrm{d}_{1}, 500 \mathrm{MHz}\right) \delta(\mathrm{ppm}): 10.29(\mathrm{~s}, 1 \mathrm{H}), 9.92$ (s, $1 \mathrm{H}), 8.00-7.98(\mathrm{~m}, 2 \mathrm{H}), 7.86-7.84(\mathrm{~m}, 2 \mathrm{H}), 7.36-7.31(\mathrm{~m}, 5 \mathrm{H})$, 7.28-7.22 (m, $2 \mathrm{H})$, 7.06-7.04 (m, $2 \mathrm{H}), 6.97-6.95$ (m, $3 \mathrm{H}), 6.04-6.02$ (m, $1 \mathrm{H}), 5.95-5.92(\mathrm{~m}, 1 \mathrm{H}), 4.03-4.02(\mathrm{~m}, 4 \mathrm{H}), 3.79-3.76(\mathrm{~m}, 1 \mathrm{H})$, 2.70-2.67 (m, $4 \mathrm{H}), 1.68-1.67$ (m, $2 \mathrm{H}), 1.46-1.24(\mathrm{~m}, 22 \mathrm{H}) .{ }^{13} \mathrm{C} \mathrm{NMR}$
(DMSO-d $6125 \mathrm{MHz}) \delta$ (ppm): 176.1, 171.9, 171.7, 155.7, 142.8, $141.5,141.2,134.8,128.6,125.2,122.5,122.4,122.2,122.0,114.3$, $110.0,98.9,49.2,44.9,39.2,28.4,28.3,28.0,27.9,24.4,24.3,22.5$, 21.1, 12.2, 8.6. MS (ESI ${ }^{+}$): $m /{ }_{2} \mathrm{C}_{45} \mathrm{H}_{53} \mathrm{~N}_{4} \mathrm{O}_{4}{ }^{+}$calcd. 713.4061 , found $\left[\mathrm{M}^{+}\right] 713.5278$.

\subsection{Cell models}

RLE-6TN cells were cultivated in DMEM medium under a $5 \% \mathrm{CO}_{2}$ atmosphere at $37^{\circ} \mathrm{C}$. Pulmonary fibrosis cell models were established using living cells by treating with $50 \mu \mathrm{M}$ transforming growth factor beta 1 (TGF- $\beta 1$ ) for $72 \mathrm{~h}$. The pulmonary fibrosis cell models and normal cells were treated with Cy-GGT at $37^{\circ} \mathrm{C}$ for $30 \mathrm{~min}$. As oxidative stress model, living cells were stimulated with paraquat $(50 \mu \mathrm{M})$ to induce excessive production of $\mathrm{O}_{2}{ }^{\cdot-}$ for another $8 \mathrm{~h}$ at $37^{\circ} \mathrm{C}$, that could cause severe oxidative stress level. Prior to imaging, living cells were cultivated with Cy-GGT at $37^{\circ} \mathrm{C}$ for $30 \mathrm{~min}$. With a commercial probe (DHE), we examined the oxidative stress level stimulated by paraquat. We implemented cell imaging experiments with RLE-6TN cells cultivated with DHE at $37^{\circ} \mathrm{C}$ for $30 \mathrm{~min}$.

\subsection{Cytotoxicity of Cy-GGT}

We determined the toxicity of Cy-GGT to cells by MTT method. First, we seeded RLE-6TN cells in good condition on 96-well plates for a whole day. Subsequently, the RLE-6TN cells were administrated with diverse concentrations of Cy-GGT $(0.1,1,10$ and $100 \mu \mathrm{M})$ in an atmosphere of $5 \% \mathrm{CO}_{2}$ for a whole day. The cells were then cultured under an atmosphere of $5 \% \mathrm{CO}_{2}$ for another $4 \mathrm{~h}$, after adding MTT ( $5 \mathrm{mg} / \mathrm{mL}$ ) to each well. Next, formazan crystal dissolved in DMSO $(150 \mu \mathrm{L})$ formed. The absorbance was assessed at $570 \mathrm{~nm}$. The above data are the average standard deviation of 5 independent tests.

\subsection{Animal models}

These C57BL/ 6 mice purchased from the Model Animal Research Center (Nanjing University) for 8 weeks were raised in groups. They freely accessed to enough water and food, and lived in a 12:12 lightdark cycle. To establish the mice model of pulmonary fibrosis, we instilled bleomycin $(5 \mathrm{mg} / \mathrm{kg})$, which is dissolvable in physiological saline, into the trachea under anesthesia. The drug was infused only once, and the mice were raised for 28 days.

\subsection{In vivo imaging in living mice}

Mice model was established as described in the chapter of "Animal model". Then all the C57BL/ 6 mice were administered an intratracheal instillation of Cy-GGT $(10 \mu \mathrm{M}, 10 \mu \mathrm{L}$ in 1:99 DMSO/saline v/v). Prior to imaging, we anesthetized all C57BL/ 6 mice by injection with $4 \%$ chloral hydrate $(0.25 \mathrm{~mL})$. The imaging of living mice were obtained by In Vivo Imaging System. T he above data were the mean standard deviation of 5 independent tests. In addition, in order to clearly show the reaction sites of $\mathrm{C} 57 \mathrm{BL} / 6$ mice, the fluorescence image and the bright field image were merged.

\section{Results and discussion}

\subsection{Design strategies of $C y-G G T$}

The synthetic approaches of probe Cy-GGT was exhibited in Scheme S1. These detailed procedures of the mixtures were described in Supporting Information. These compounds in this part were characterized by HRMS, ${ }^{1} \mathrm{H}$ NMR and ${ }^{13} \mathrm{C}$ NMR. We applied a heptamethine cyanine dye as the fluorophore unit located in the near-infrared region to emit the detection signal. The optical imaging with NIR fluorophores has higher tissue penetration depth and sensitivity, because the tissue 


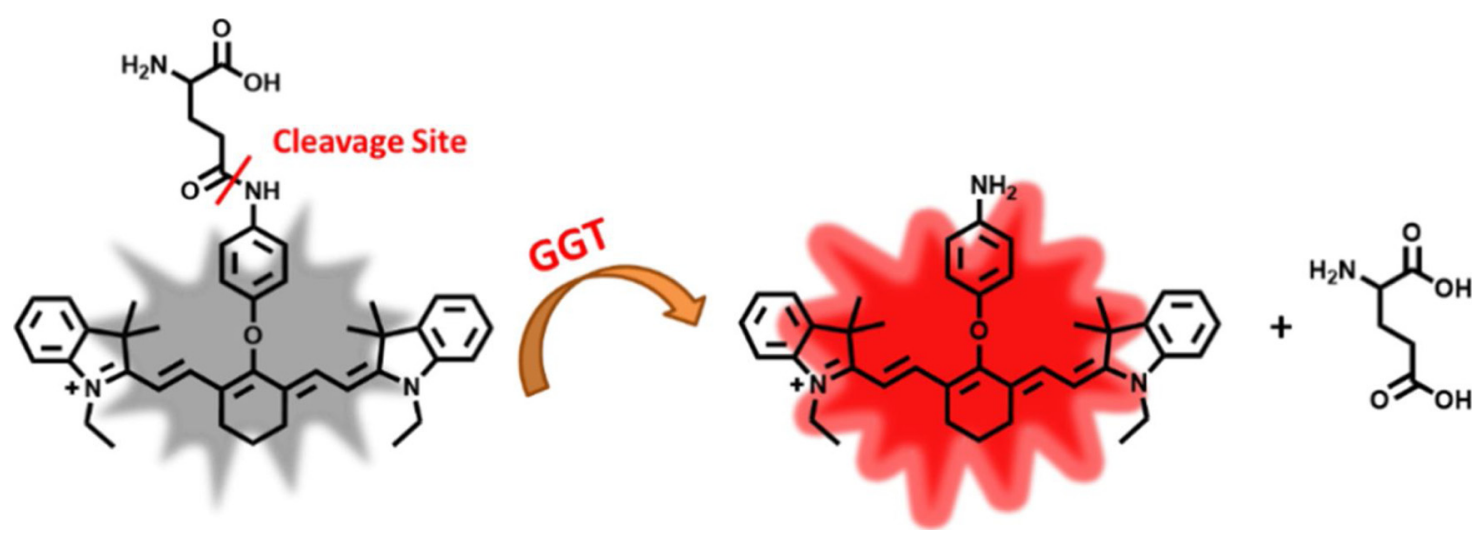

Scheme 1. The response mechanism for the probe towards GGT.

auto-fluorescence and absorption are greatly low in the NIR spectral range. In previous experiments, we incorporated the specific recognition moiety of the $\gamma$-glutamyl group into fluorophore unit to get a probe for detecting GGT. Interestingly, a $\gamma$-glutamyl amide group acts as an enzyme active trigger of GGT for its high sensitivity and characteristic response. For establishing our ideal probe, the Boc-protected glutamic acid moiety was grafted onto the fluorescence reporter, following deprotection under the acidic condition (Scheme S1). Therefore, the electron-withdrawing glutamyl amide moiety could quench the fluorescent signals of Cy-GGT. The enzyme-triggered cleavage reaction stimulated by GGT led to the release of electron-donating amine moiety, thereby acquiring a "turn-on" fluorescent response with a long emission wavelength $(780 \mathrm{~nm})$. The described detection mechanism was exhibited in Scheme 1. We could use this probe to track the change of GGT in cells and in vivo. Additionally, the physiological function of GGT in occurrence and development of IPF would be excavated.

\subsection{Spectroscopic properties}

We studied the absorption spectra and fluorescence spectral response of Cy-GGT to GGT in HEPES buffer solution (10 mM, pH = 7.4). The maximum absorption wavelength of Cy-GGT $(10 \mu \mathrm{M})$ locates in $770 \mathrm{~nm}$ with $\varepsilon=2.6 \times 10^{4} \mathrm{M}^{-1} \mathrm{~cm}^{-1}$ (Fig. S3). As we expected, CyGGT showed a remarkable enhancement of absorption peak at the wavelength of $770 \mathrm{~nm}$ after adding $50 \mathrm{U} / \mathrm{L}$ GGT. For further exploring the specific fluorescence response of Cy-GGT to GGT, the fluorescence spectra of Cy-GGT were checked. The experiment results indicated that the addition of GGT (from 0 to $100 \mathrm{U} / \mathrm{L}$ ) induced the increase of fluorescence signals, which centered at $780 \mathrm{~nm}$ (Fig. 1a). Thus, we quantified the fluorescence response of Cy-GGT to GGT with the wavelength at $780 \mathrm{~nm}$ as the suitable fluorescence signals. It was noteworthy that the low fluorescence intensity of CyMito was attributed to d-PET process $(\Phi C y-M i t o=0.015)$. Cy-GGT has the low fluorescence quantum yields $\left(\Phi_{\mathrm{Cy}-\mathrm{GGT}}=0.025\right)$. After Cy-GGT was treated with increasing concentrations of GGT (0-100 U/L), the fluorescence quantum yields was $\Phi_{\mathrm{Cy}-\mathrm{GGT}}+\mathrm{GGT}=0.13$. The light stability of our probe is an important factor to be evaluated, therefore, the curve of fluorescence intensity of Cy-GGT + GGT upon continuous laser irradiation for $35 \mathrm{~min}$ was shown in Fig. S5. The fluorescence intensity visibly decreased within $35 \mathrm{~min}$ irradiation. The calibration curve was exhibited in Fig. 1b, and an excellent linearity with the GGT concentrations ranging from 0 to $100 \mathrm{U} / \mathrm{L}$ was obtained. The regression equation was $F_{780 \mathrm{~nm}}=8.2641[\mathrm{GGT}](\mathrm{U} / \mathrm{L})+18.3950(r=0.9960)$. Under the existing test conditions, the limit of detection ( $3 \sigma /$ slope, where $\sigma$ is the standard deviation of blank measurements, $n=11$ ) towards GGT was calculated to be $7.6 \mathrm{mU} / \mathrm{L}$. The result illustrated that Cy-GGT had higher sensitivity to GGT. The probe Cy-GGT has the potential to be applied to various biological detections.

\subsection{Kinetic responds of Cy-GGT against GGT}

The fluorescence signal had almost no change in the $\mathrm{pH}$ range from 4.0 to 9.0. After the probe Cy-GGT reacted with GGT, the fluorescence signal was significantly enhanced especially maintained a maximum intensity at $\mathrm{pH} 7.4$, indicating that Cy-GGT responded well to GGT under simulated physiological conditions. Furthermore, the reaction kinetic was explored by the time-dependent fluorescence response (Fig. 1c). We selected the reaction time of $50 \mathrm{~min}$ for the present system. Moreover, in the absence of GGT, there was no obvious change in the fluorescence of the probe Cy-GGT within $50 \mathrm{~min}$. After GGT was added, the fluorescence signals gradually increased to its maximum within about $30 \mathrm{~min}$. The second-order rate constant $\left(\mathrm{k}_{2}\right)$ for reaction between Cy-GGT and GGT is $1.09 \times 10^{7} \mathrm{M}^{-1} \mathrm{~s}^{-1}$. And higher GGT concentrations led to faster reaction velocity and stronger fluorescence generation. These aforementioned results demonstrated that Cy-GGT was able to respond to GGT quickly. The fast reaction kinetics would facilitate real-time detection of GGT in complex organisms.

\subsection{Selectivity of probe towards GGT}

Next, we assessed the fluorescent response of Cy-GGT to other common physiologically relevant species. As shown in Fig. S4, Cy-GGT was treated with diverse biospecies, including tocopherols, S-nitrosoglutathione (GSNO), NaHS, $\mathrm{Na}_{2} \mathrm{~S}_{4}$, L-cysteine (L-cys), L-arginine (Larg), ascorbic acid, glutathione (GSH) and tyrosine. The experiment data distinctly demonstrated that only GGT could significantly increase the fluorescence emission (Fig. S4). More importantly, the analogous functional enzymes (nitroreductase, glutathione S-transferases, horseradish peroxidase, alkaline phosphatase, monoamine oxidase $A, \beta$-galactosidase and monoamine oxidase B also could not result in any influence in the experiments (Fig. 1d). These results certified that Cy-GGT exhibited great selectivity to GGT in the presence of diverse biologically relevant species.

\subsection{Imaging of GGT in RLE-6TN cells}

Owing to the outstanding ability of Cy-GGT to track GGT specifically and sensitively, the potential application of Cy-GGT for detecting GGT in living cells is next explored. The RLE-6TN cells were chosen as test models to explore the applicability of Cy-GGT in cells. Because the safety of Cy-GGT is extremely vital for biological application, we first employed MTT to detect the cytotoxicity of the probe. As shown in Fig. 2d, Cy-GGT is less cytotoxic to living cells, as evidenced by high cell survival rates under normal physiological conditions. To prepare for fluorescence imaging, all three groups of cells need to be incubated with Cy-GGT at $37^{\circ} \mathrm{C}$ for $30 \mathrm{~min}$. Laser scanning confocal microscopy was utilized to perform cell imaging experiments, which were further tested by flow cytometry analysis. The first cell groups were the control. 
a

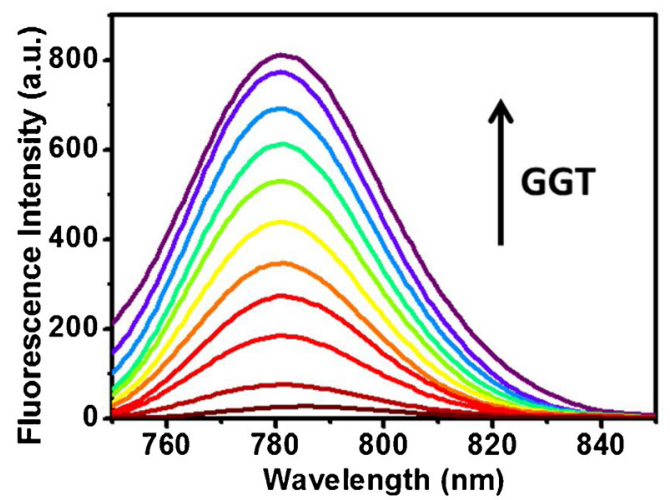

C

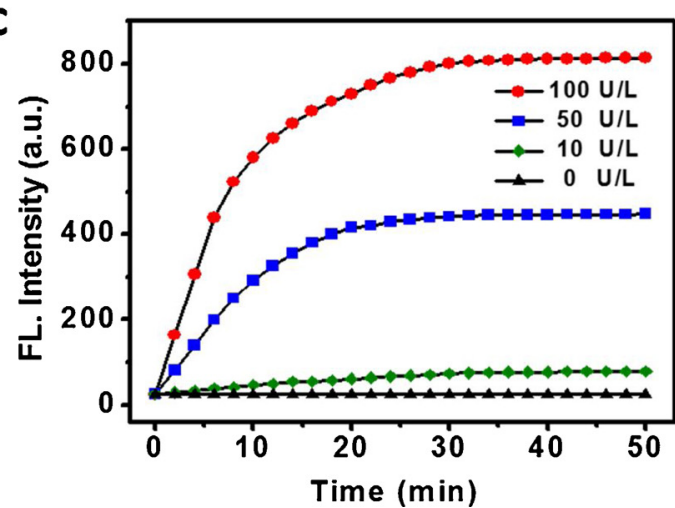

b

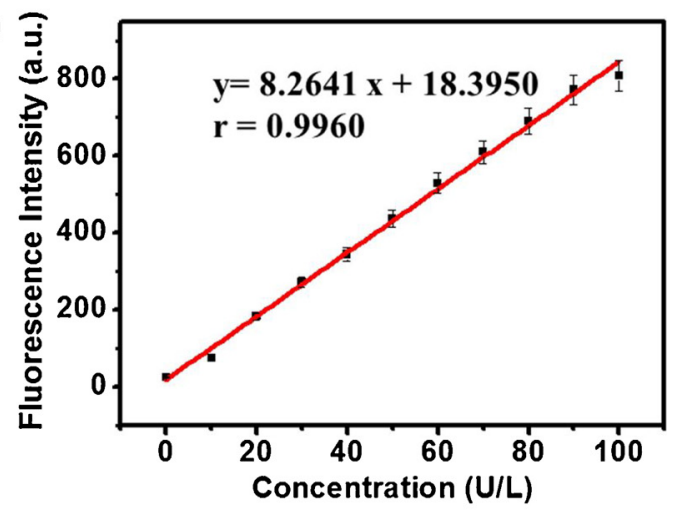

d

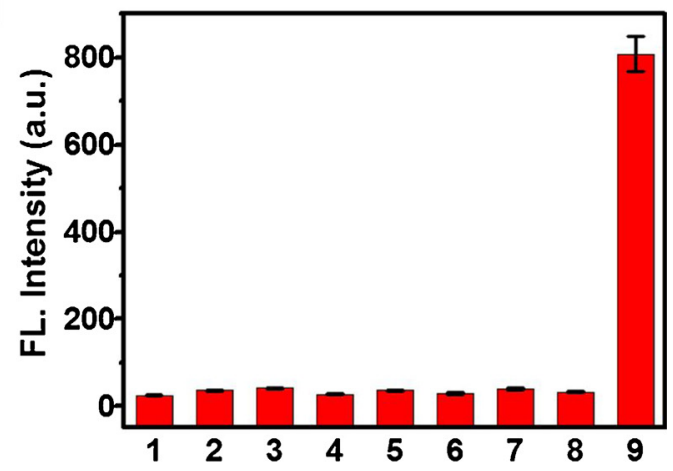

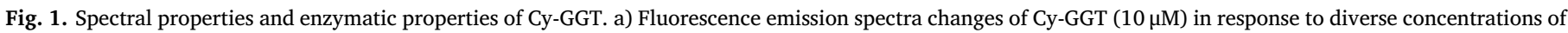

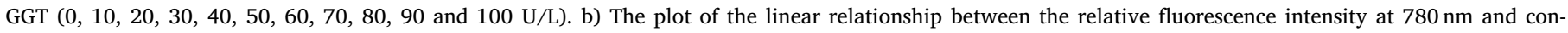

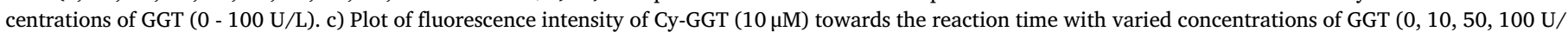

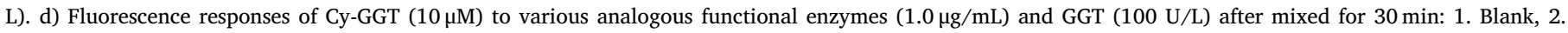

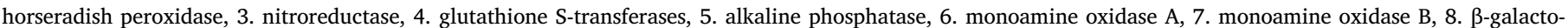
sidase, 9. GGT. The tests were carried out at $37^{\circ} \mathrm{C}$ in HEPES buffer solution (10 mM, pH 7.4).

The commercial inhibitor of GGT, GGsTop (1 mM), was added to RLE$6 \mathrm{TN}$ cells in the second group for $24 \mathrm{~h}$ before fluorescence imaging, which reduced intracellular GGT concentrations. The third group of RLE-6TN cells overexpressed GGT by transfecting GGT-RNA. The fluorescence intensity of RLE-6TN cells in Fig. 2a gradually increased during the testing process. The rather lower fluorescence intensity was offered in Fig. 2b, because the GGT activity was inhibited by GGsTop. The fluorescence intensity of Fig. 2c was stronger than that in Fig. 2a, revealing the higher level of GGT in these transfected cells. Additionally, the correctness of the above results was verified again by flow cytometry analysis (Fig. 2a-2c). The activity of GGT in the three groups was further determined via GGT activity assay kit (Fig. 2e), which is concordant with the data gained from flow cytometry analysis and fluorescence image. The average fluorescence intensity was displayed in Fig. 2f for directly quantifying GGT. The function of probe $\mathrm{Cy}$ GGT for sensitive imaging GGT in living cells was illustrated by the above experiments, and we could investigate the essential role of GGT in physiological and pathological processes with Cy-GGT as a serviceable and promising tool.

\subsection{Evaluation of the changes of GGT in the cell models of pulmonary fibrosis}

Higher level of oxidative stress in IPF could lead to increased GGT $[55,56]$. Hence, prior to exploring the connection between pulmonary fibrosis and GGT, the relationship between oxidative stress and GGT was first validated. We applied Cy-GGT to monitor the changes of GGT under oxidative stress condition. The RLE-6TN cells in Fig. 3a were stimulated with paraquat $(50 \mu \mathrm{M})$ for $8 \mathrm{~h}$ to trigger the excessive production of $\mathrm{O}_{2}{ }^{\cdot-}$, leading to cell damage under oxidative stress state. We next treated the RLE-6TN cells with Cy-GGT at $37^{\circ} \mathrm{C}$ for $30 \mathrm{~min}$ and then imaging. Dihydroethidium (DHE), a commercial fluorescent probe, was further utilized to monitor the oxidative stress condition. As displayed in Fig. 3b, the RLE-6TN cells offered a strong fluorescent intensity in the green channel, which revealed that the irritation stimulated by paraquat would result in a grievous oxidative stress condition. The intense fluorescence intensity in red channel was exhibited in RLE6TN cells stimulated by paraquat as expected, disclosing the fact that more GGT were generated (Fig. 3a). We applied the flow cytometry analysis to verify the data receiving from fluorescence imaging of cells (Fig. 3a and b). The average fluorescence intensity of RLE-6TN cells imaging was displayed in Fig. 3c. Then the fluctuations of GGT activity were examined via a commercial GGT activity assay kit (Fig. 3d). This result indicated that the function of our probe was consistent with the commercial kit. The distinct increase of intracellular GGT in living cells cultivated with oxidative stress condition was amply confirmed by the above results, probably because GGT could regulate the oxidative stress generated under pathological conditions.

The unknown relationship between GGT and pulmonary fibrosis was inspected based on the fact that the GGT level in cells elevated under oxidative stress state. For establishing pulmonary fibrosis cell models successfully, RLE-6TN cells were stimulated with TGF- $\beta 1$ $(50 \mu \mathrm{M})$ for $72 \mathrm{~h}$. As displayed in Fig. 3e, the RLE-6TN cells cultured with Cy-GGT for $30 \mathrm{~min}$ were selected as control. A higher fluorescence intensity in the pulmonary fibrosis cell models than in the control group clarified a higher GGT level in these cells. Furthermore, we employed the flow cytometry analysis to confirm the data receiving from imaging of cells (Fig. 3e). The average of GGT activity were further determined via GGT activity assay kit (Fig. 3g). One of the typical manifestation in pulmonary fibrosis is the phenomena of mesenchymal characteristics, 

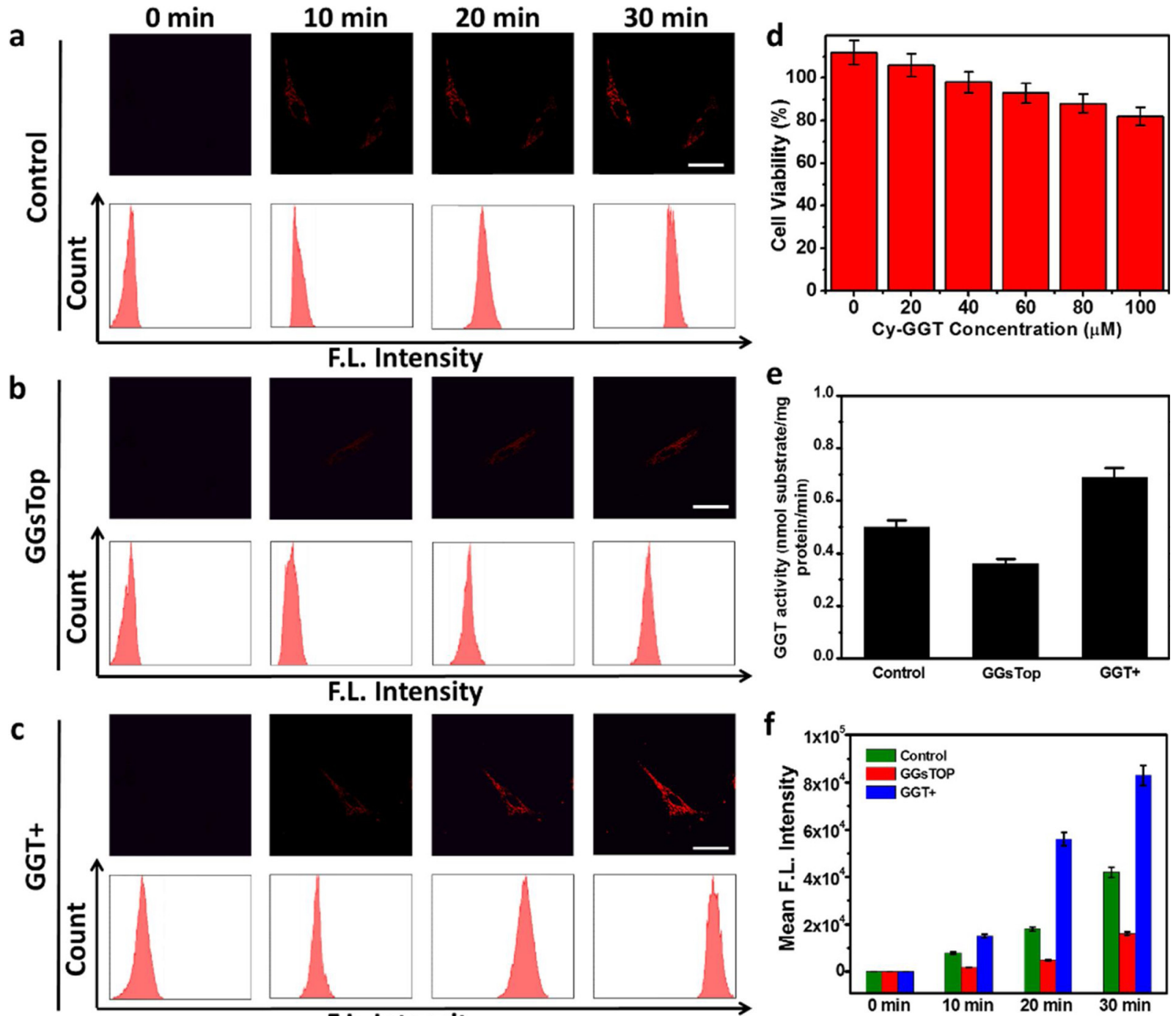

\section{F.L. Intensity}
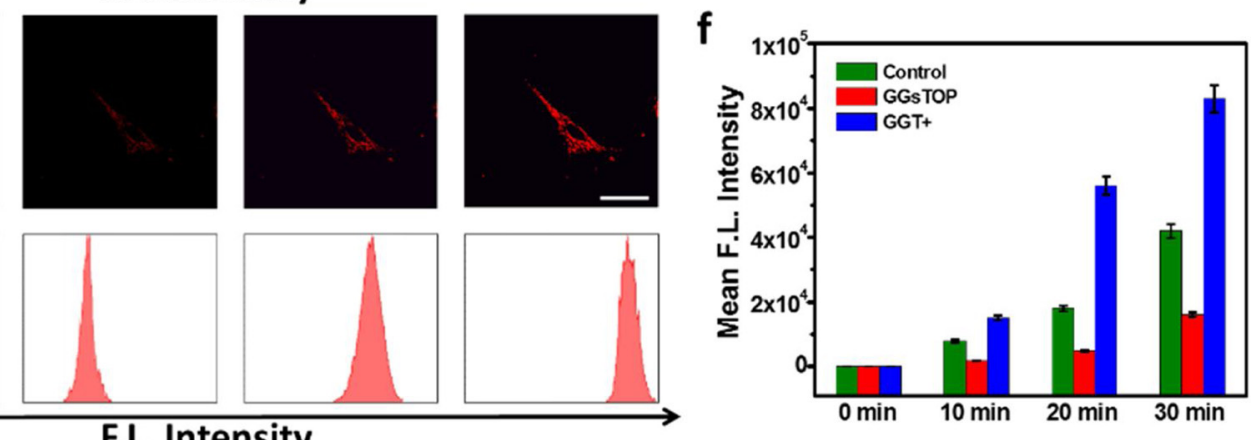

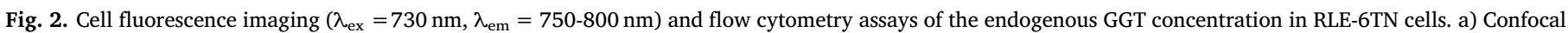

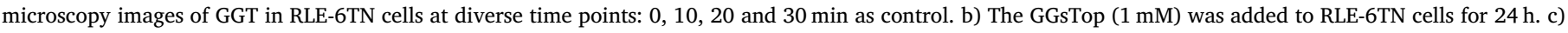

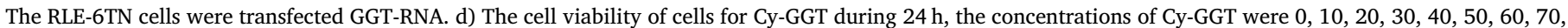

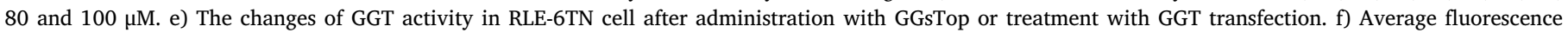
intensities of images in (a-c). Images are representative of $n=5$ independent tests. Scale bars: $20 \mu \mathrm{m}$.

involving the loss of E-cadherin protein (E-cadherin) as a marker for epithelial cells, and the abnormal performance of mesenchymal markers, like transcription repressor snail, vimentin, and alpha-smooth muscle actin protein ( $\alpha$-SMA) in lung cells [57]. The $\alpha$-SMA and Ecadherin are widely utilized as effective indicators to verify the severity of pulmonary fibrosis. Western blot (WB) was performed to assess $\alpha$ SMA and E-cadherin concentration changes. As shown in Fig. 3h, in the cell model of pulmonary fibrosis, the increase of $\alpha$-SMA and the decrease of E-cadherin certified that the cells stimulated with TGF- $\beta 1$ were much more severe than the control group cells. The relative protein expressions of $\alpha$-SMA and E-cadherin in Fig. 3i were consistent with Fig. $3 \mathrm{~h}$. The above results verified that the pulmonary fibrosis cell models were successfully established, and the level of GGT in the cell model of pulmonary fibrosis was higher than that of normal cells. As our expectations, the intracellular GGT concentration was closely connected to the extent of pulmonary fibrosis, and the GGT level was potential to instruct the process of pulmonary fibrosis.
3.7. Evaluation of the changes of GGT in the mice models of pulmonary fibrosis

We next performed trials to investigate the potential application of Cy-GGT as an effective imaging tool in the mice models of pulmonary fibrosis. The living C57Bl/6 mice were stimulated with bleomycin to establish pulmonary fibrosis mice models. We performed H\&E and Masson staining of lung tissues in each group to verify the success of the model. Cy-GGT was intratracheal administration for $1 \mathrm{~h}$ before fluorescence imaging in vivo. We applied an in vivo imaging system to measure the GGT in different testing groups. The increase of fluorescence intensity in the mice models of pulmonary fibrosis indicated the content of GGT elevated with the aggravation of pulmonary fibrosis (Fig. 4a). As shown in Fig. 4b, only the ex vitro imaging of lung showed enhanced fluorescence clearly, and there were no significant fluorescence changes in other organs, involving the heart, spleen, liver, and kidneys. The average fluorescence intensities of every group were displayed in Fig. 4d. H\&E and Masson staining were employed to verify the successful establishment of pulmonary fibrosis model and degree of pulmonary fibrosis (Fig. 4c). These in vivo results clarified intimate 

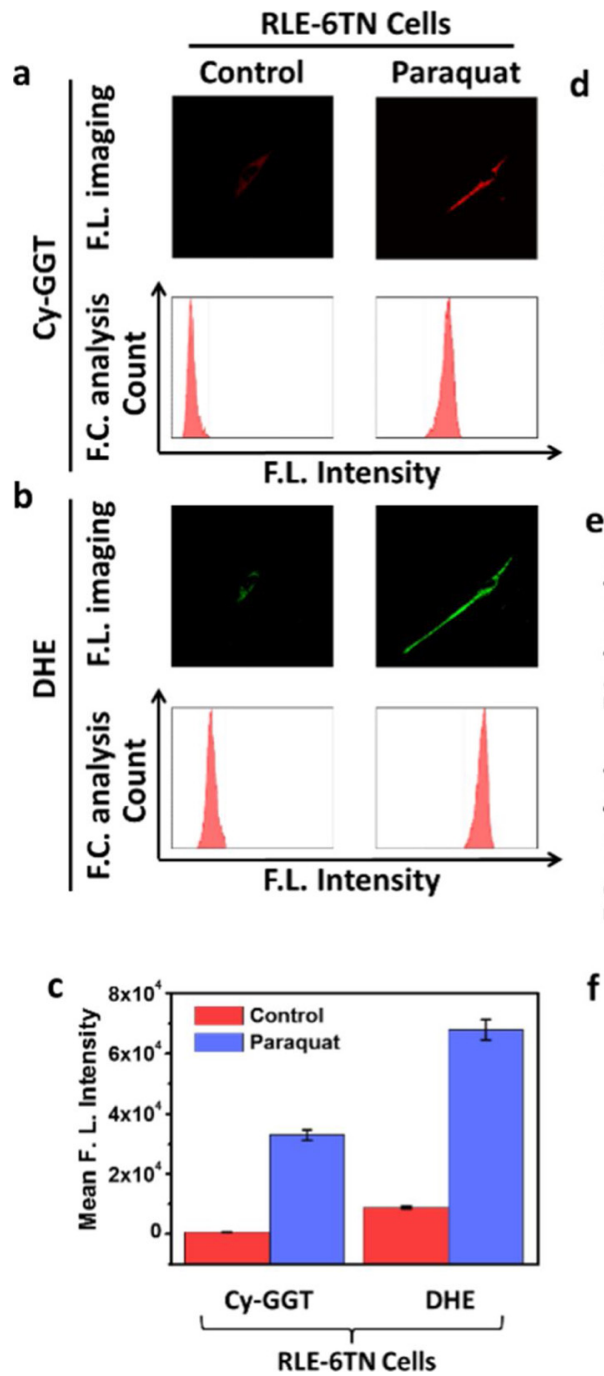

d

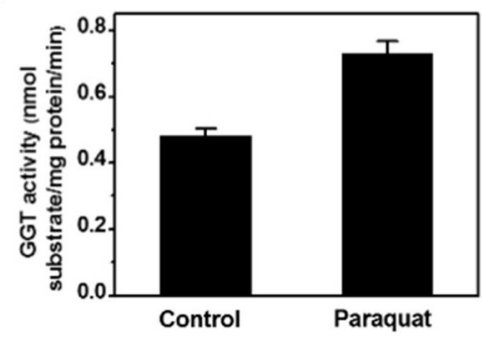

RLE-6TN Cells
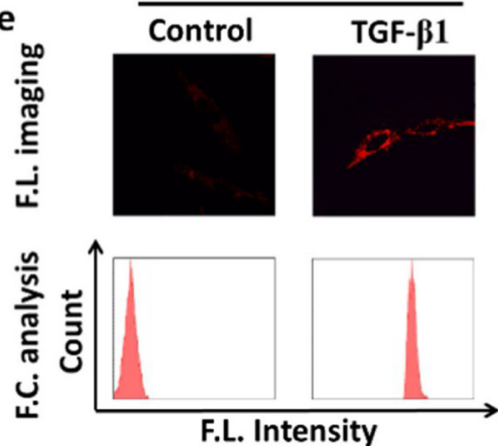

f

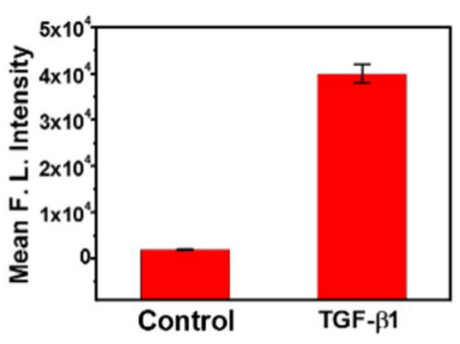

g

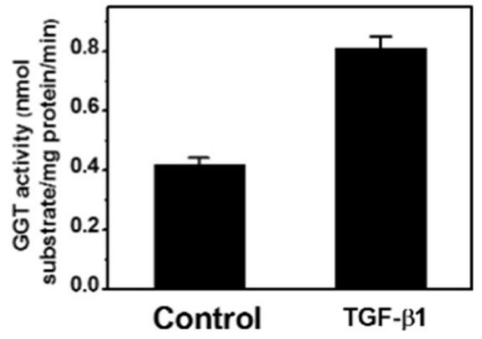

h

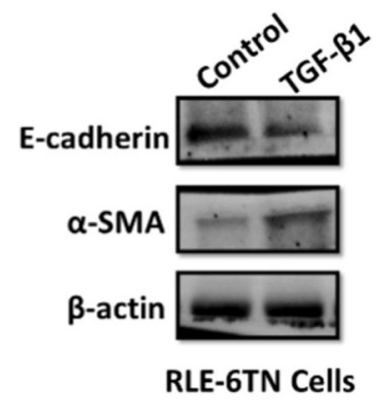

i

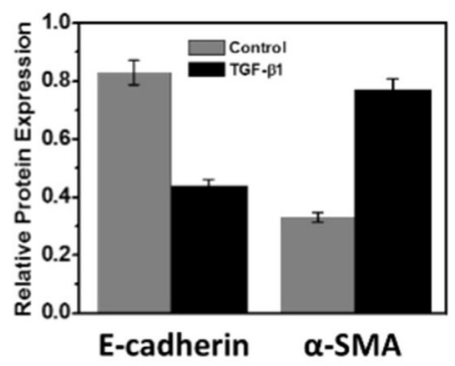

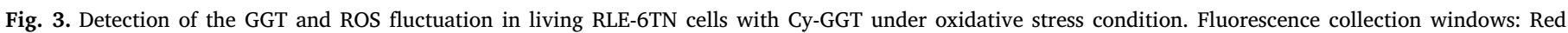

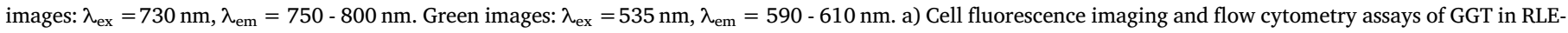

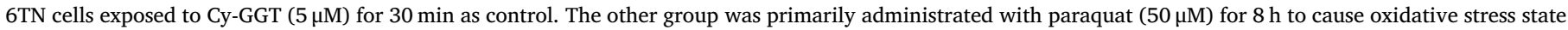

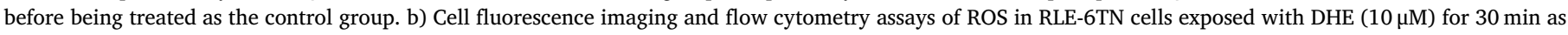

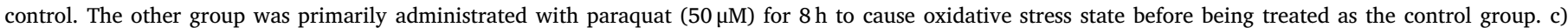

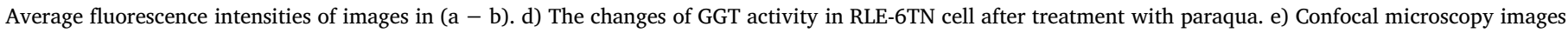

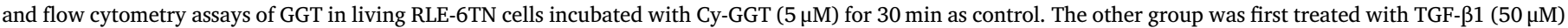

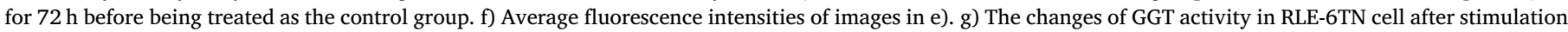

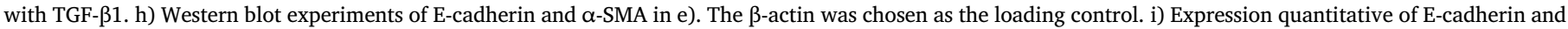

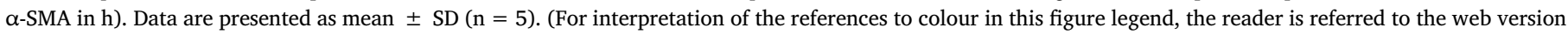
of this article).

association between GGT and pulmonary fibrosis, GGT may play a crucial part in pathophysiology of IPF. More importantly, our probe has great potential as an effective tool to monitor the development of pulmonary fibrosis in actual mice models.

\section{Conclusion}

In this study, we design a NIR fluorescent probe Cy-GGT, as an outstanding and rapid tool, for detecting endogenous GGT in living cells and in vivo. With the help of Cy-GGT, we successfully detect the changes of GGT in oxidative stress cell models and in pulmonary fibrosis cell models. Compared with normal cells, the GGT level in the cell models of oxidative stress and pulmonary fibrosis is higher. To further confirm the underlying association between GGT level and pulmonary fibrosis degree, we validate the GGT increased in the mice models of pulmonary fibrosis. The effective application of the probe in the mice models also demonstrated its ability to imaging GGT in vivo due to its NIR emission. The results suggest GGT is inextricably linked to the occurrence and development of pulmonary fibrosis, GGT might be a promising marker for IPF diagnosis. We anticipate that our probe, as a practical and accurate detection tool, has promising applications in the precise diagnosis of IPF in clinical.

\section{Funding}

The authors declare no competing financial interest.

\section{CRediT authorship contribution statement}

Na He: Conceptualization, Validation, Formal analysis, Investigation, Writing - original draft. Yude Wang: Data curation, Methodology, Investigation, Software. Yan Huang: Conceptualization, 
a

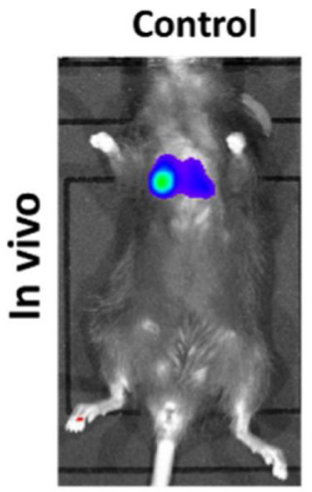

C
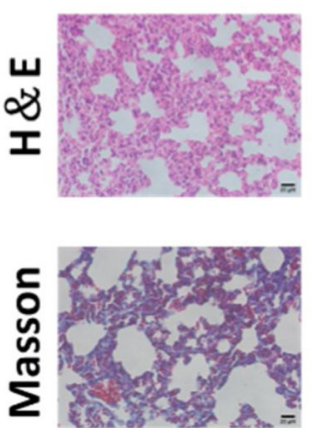

Bleomycin
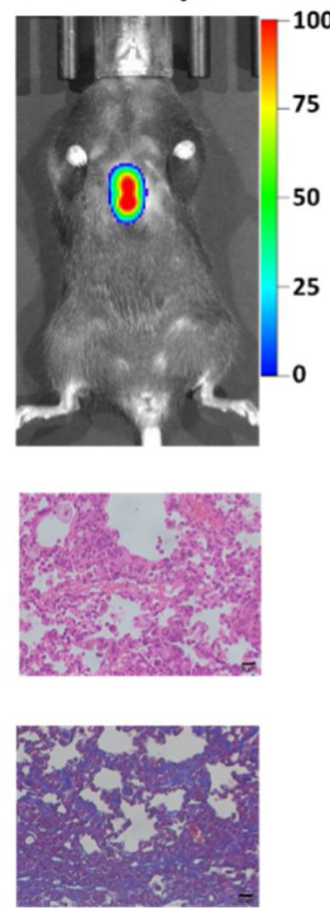

b Heart Liver Spleen Lung Kidney
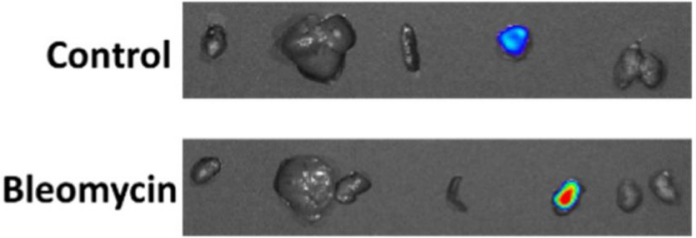

d

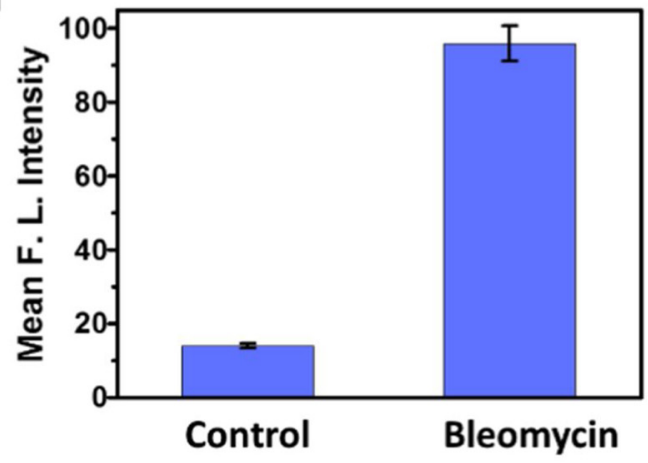

Fig. 4. Fluorescence images in pulmonary fibrosis mice $\left(\lambda_{\text {ex }}=730 \mathrm{~nm}, \lambda_{\text {em }}=750-800 \mathrm{~nm}\right)$. a) Images of GGT utilized Cy-GGT (100 $\mu \mathrm{M}, 50 \mu \mathrm{L}$ in $1: 99 \mathrm{DMSO} / \mathrm{saline}$, $\mathrm{v} / \mathrm{v}$ ) of each group, normal mice as control group; mice with bleomycin stimulation 28 days as bleomycin group. Prior to imaging in vivo, the mice models in these experiments were intratracheally administered with Cy-GGT for $30 \mathrm{~min}$. b) Images of isolated organs, involving heart, liver, spleen, lung, and kidney. c) H\&E and Masson pathology of each group. d) Mean fluorescence intensities of images in a). Data are presented as mean \pm SD $(n=5)$.

Software, Visualization, Writing - review \& editing. Xiaoyan Wang: Validation, Formal analysis, Investigation, Validation. Lingxin Chen: Conceptualization, Funding acquisition, Methodology, Writing - review \& editing. Changjun Lv: Funding acquisition, Conceptualization, Methodology, Writing - review \& editing.

\section{Declaration of Competing Interest}

The authors declare that they have no known competing financial interests or personal relationships that could have appeared to influence the work reported in this paper.

\section{Acknowledgments}

This work was supported by the National Nature Science Foundation of China (Nos. 81573393, 21804010, 21976209, 81870001), the science and technology innovation development plan of Yantai of China (No. 2020MSGY113), the Research Initiation Fund of Binzhou Medical University (Grant No. BY2019KYQD39, BY2020KYQD01) and Taishan Scholar Project Special Funding (No. ts20190962).

\section{Appendix A. Supplementary data}

Supplementary material related to this article can be found, in the online version, at doi:https://doi.org/10.1016/j.snb.2020.128565.

\section{References}

[1] R.H. Zou, D.J. Kass, K.F. Gibson, K.O. Lindell, The role of palliative care in reducing symptoms and improving quality of life for patients with idiopathic pulmonary fibrosis: a review, Pulm. Ther. 1 (2020) 35-46.

[2] M. Lv, Y. Liu, S. Ma, Z. Yu, Current advances in idiopathic pulmonary fibrosis: the pathogenesis, therapeutic strategies and candidate molecules, Future Med. Chem. 19 (2019) 2595-2620.
[3] T.J. King, W.Z. Bradford, S. Castro-Bernardini, E.A. Fagan, I. Glaspole, M.K. Glassberg, E. Gorina, P.M. Hopkins, D. Kardatzke, L. Lancaster, D.J. Lederer, S.D. Nathan, C.A. Pereira, S.A. Sahn, R. Sussman, J.J. Swigris, P.W. Noble, A phase 3 trial of pirfenidone in patients with idiopathic pulmonary fibrosis, N. Engl. J. Med. 22 (2014) 2083-2092.

[4] L. Richeldi, R.M. du Bois, G. Raghu, A. Azuma, K.K. Brown, U. Costabel, V. Cottin, K.R. Flaherty, D.M. Hansell, Y. Inoue, D.S. Kim, M. Kolb, A.G. Nicholson,

P.W. Noble, M. Selman, H. Taniguchi, M. Brun, F. Le Maulf, M. Girard, S. Stowasser, R. Schlenker-Herceg, B. Disse, H.R. Collard, Efficacy and safety of nintedanib in idiopathic pulmonary fibrosis, N. Engl. J. Med. 22 (2014) 2071-2082.

[5] J. Baddini-Martinez, B.G. Baldi, C.H. Costa, S. Jezler, M.S. Lima, R. Rufino, Update on diagnosis and treatment of idiopathic pulmonary fibrosis, J. Bras. Pneumol. 5 (2015) 454-466.

[6] A.M. Cantin, S.L. North, G.A. Fells, R.C. Hubbard, R.G. Crystal, Oxidant-mediated epithelial cell injury in idiopathic pulmonary fibrosis, J. Clin. Invest. 6 (1987) 1665-1673.

[7] L. Hecker, R. Vittal, T. Jones, R. Jagirdar, T.R. Luckhardt, J.C. Horowitz, S. Pennathur, F.J. Martinez, V.J. Thannickal, NADPH oxidase-4 mediates myofibroblast activation and fibrogenic responses to lung injury, Nat. Med. 9 (2009) 1077-1081.

[8] L.M. De Young, W.L. Richards, W. Bonzelet, L.L. Tsai, R.K. Boutwell, Localization and significance of gamma-glutamyltranspeptidase in normal and neoplastic mouse skin, Cancer Res. 11 (Pt 1) (1978) 3697-3701.

[9] H. Shinozuka, M.A. Sells, S.L. Katyal, S. Sell, B. Lombardi, Effects of a cholinedevoid diet on the emergence of gamma-glutamyltranspeptidase-positive foci in the liver of carcinogen-treated rats, Cancer Res. 7 (Pt 1) (1979) 2515-2521.

[10] C. Di Ilio, G. Polidoro, A. Arduini, A. Muccini, G. Federici, Glutathione peroxidase, glutathione reductase, glutathione S-transferase, and gamma-glutamyltranspeptidase activities in the human early pregnancy placenta, Biochem. Med. 2 (1983) 143-148.

[11] M.S. Rao, M. Tatematsu, V. Subbarao, N. Ito, J.K. Reddy, Analysis of peroxisome proliferator-induced preneoplastic and neoplastic lesions of rat liver for placental form of glutathione S-transferase and gamma-glutamyltranspeptidase, Cancer Res. 10 (1986) 5287-5290.

[12] M.H. Hanigan, W.A. Ricketts, Extracellular glutathione is a source of cysteine for cells that express gamma-glutamyl transpeptidase, Biochemistry-US 24 (1993) 6302-6306.

[13] A. Pompella, V. De Tata, A. Paolicchi, F. Zunino, Expression of gamma-glutamyltransferase in cancer cells and its significance in drug resistance, Biochem. Pharmacol. 3 (2006) 231-238.

[14] A.M. Strasak, G. Goebel, H. Concin, R.M. Pfeiffer, L.J. Brant, G. Nagel, W. Oberaigner, N. Concin, G. Diem, E. Ruttmann, U. Gruber-Moesenbacher, F. Offner, A. Pompella, K.P. Pfeiffer, H. Ulmer, Prospective study of the association of serum gamma-glutamyltransferase with cervical intraepithelial neoplasia III and invasive cervical cancer, Cancer Res. 9 (2010) 3586-3593.

[15] D.H. Lee, M.D. Gross, D.J. Jacobs, Association of serum carotenoids and tocopherols with gamma-glutamyltransferase: the Cardiovascular Risk Development in Young 
Adults (CARDIA) Study, Clin. Chem. 3 (2004) 582-588.

[16] C. Ravuri, G. Svineng, S. Pankiv, N.E. Huseby, Endogenous production of reactive oxygen species by the NADPH oxidase complexes is a determinant of gamma-glutamyltransferase expression, Free Radic. Res. 5 (2011) 600-610.

[17] D.R. Karp, K. Shimooku, P.E. Lipsky, Expression of gamma-glutamyl transpeptidase protects ramos B cells from oxidation-induced cell death, J. Biol. Chem. 6 (2001) 3798-3804.

[18] S. Pandur, S. Pankiv, M. Johannessen, U. Moens, N.E. Huseby, Gamma-glutamyltransferase is upregulated after oxidative stress through the Ras signal transduction pathway in rat colon carcinoma cells, Free Radic. Res. 12 (2007) 1376-1384.

[19] A. Pardo, V. Ruiz, J.L. Arreola, R. Ramirez, J. Cisneros-Lira, M. Gaxiola, R. Barrios, S.V. Kala, M.W. Lieberman, M. Selman, Bleomycin-induced pulmonary fibrosis is attenuated in gamma-glutamyl transpeptidase-deficient mice, Am. J. Respir. Crit. Care Med. 6 (2003) 925-932.

[20] M. Orlowski, A. Meister, Gamma-glutamyl-P-nitroanilide: a new convenient substrate for determination and study of 1- and D-gamma-glutamyltranspeptidase activities, Biochim. Biophys. Acta (1963) 679-681.

[21] K. Kiuchi, K. Kiuchi, T. Nagatsu, A. Togari, H. Kumagai, Highly sensitive assay for gamma-glutamyltranspeptidase activity by high-performance liquid chromatography with electrochemical detection, J. Chromatogr. 1 (1986) 191-198.

[22] V. Kulhanek, D.M. Dimov, A new useful modification for the determination of gamma-glutamyltranspeptidase activity, Clin. Chim. Acta 5 (1966) 619-623.

[23] S. Erbas-Cakmak, S. Kolemen, A.C. Sedgwick, T. Gunnlaugsson, T.D. James, J. Yoon, E.U. Akkaya, Molecular logic gates: the past, present and future, Chem. Soc. Rev. 7 (2018) 2228-2248.

[24] X. Chen, C.S. Lim, D. Lee, S. Lee, S.J. Park, H.M. Kim, J. Yoon, Two-photon fluorescence sensors for imaging NMDA receptors and monitoring release of $\mathrm{Zn}^{2+}$ ) from the presynaptic terminal, Biosens. Bioelectron. (2017) 770-779.

[25] B. Dong, X. Song, X. Kong, C. Wang, Y. Tang, Y. Liu, W. Lin, Simultaneous nearinfrared and two-photon in vivo imaging of $\mathrm{H}_{2} \mathrm{O}_{2}$ using a ratiometric fluorescent probe based on the unique oxidative rearrangement of oxonium, Adv. Mater. 39 (2016) 8755-8759.

[26] K. Liu, X. Kong, Y. Ma, W. Lin, Rational design of a robust fluorescent probe for the detection of endogenous carbon monoxide in living zebrafish embryos and mouse tissue, Angew. Chem. Int. Ed. Engl. 43 (2017) 13489-13492.

[27] Z. Mao, W. Feng, Z. Li, L. Zeng, W. Lv, Z. Liu, NIR in, far-red out: developing a twophoton fluorescent probe for tracking nitric oxide in deep tissue, Chem. Sci. 8 (2016) 5230-5235.

[28] Z. Lei, X. Li, X. Luo, H. He, J. Zheng, X. Qian, Y. Yang, Bright, Stable, and Biocompatible Organic Fluorophores Absorbing/Emitting in the Deep Near-Infrared Spectral Region, Angew. Chem. Int. Ed. Engl. 11 (2017) 2979-2983.

[29] Q. Xu, C.H. Heo, J.A. Kim, H.S. Lee, Y. Hu, D. Kim, K.M. Swamy, G. Kim, S.J. Nam, H.M. Kim, J. Yoon, A selective imidazoline-2-thione-bearing two-photon fluorescent probe for hypochlorous acid in Mitochondria, Anal. Chem. 12 (2016) $6615-6620$

[30] Y. Yue, F. Huo, P. Ning, Y. Zhang, J. Chao, X. Meng, C. Yin, Dual-site fluorescent probe for visualizing the metabolism of cys in living cells, J. Am. Chem. Soc. 8 (2017) 3181-3185.

[31] Wu Di, Chen Liyan, Kwon Nahyun, Juyoung Yoon, Fluorescent probes containing selenium as a guest or host, ChemUS 1 (2016) 674-698.

[32] F. Wang, G. Xu, X. Gu, Z. Wang, Z. Wang, B. Shi, C. Lu, X. Gong, C. Zhao, Realizing highly chemoselective detection of $\mathrm{H}_{2} \mathrm{~S}$ in vitro and in vivo with fluorescent probes inside core-shell silica nanoparticles, Biomaterials (2018) 82-90.

[33] H. Zhang, J. Liu, L. Wang, M. Sun, X. Yan, J. Wang, J.P. Guo, W. Guo, Amino-Sirhodamines: a new class of two-photon fluorescent dyes with intrinsic targeting ability for lysosomes, Biomaterials (2018) 10-22.

[34] J. Mei, N.L. Leung, R.T. Kwok, J.W. Lam, B.Z. Tang, Aggregation-induced emission: together we shine, united we soar!, Chem. Rev. 21 (2015) 11718-11940.

[35] D. Wu, A.C. Sedgwick, T. Gunnlaugsson, E.U. Akkaya, J. Yoon, T.D. James, Fluorescent chemosensors: the past, present and future, Chem. Soc. Rev. 23 (2017) $7105-7123$.

[36] J. Fan, M. Hu, P. Zhan, X. Peng, Energy transfer cassettes based on organic fluor ophores: construction and applications in ratiometric sensing, Chem. Soc. Rev. 1 (2013) 29-43.

[37] J.T. Hou, W.X. Ren, K. Li, J. Seo, A. Sharma, X.Q. Yu, J.S. Kim, Fluorescent bioimaging of pH: from design to applications, Chem. Soc. Rev. 8 (2017) 2076-2090.

[38] P. Verwilst, H.S. Kim, S. Kim, C. Kang, J.S. Kim, Shedding light on tau protein aggregation: the progress in developing highly selective fluorophores, Chem. Soc. Rev. 7 (2018) 2249-2265.

[39] H.W. Liu, L. Chen, C. Xu, Z. Li, H. Zhang, X.B. Zhang, W. Tan, Recent progresses in small-molecule enzymatic fluorescent probes for cancer imaging, Chem. Soc. Rev. 18 (2018) 7140-7180.

[40] J. Zhou, H. Ma, Design principles of spectroscopic probes for biological applications, Chem. Sci. 10 (2016) 6309-6315.

[41] Y. Urano, M. Sakabe, N. Kosaka, M. Ogawa, M. Mitsunaga, D. Asanuma, M. Kamiya, M.R. Young, T. Nagano, P.L. Choyke, H. Kobayashi, Rapid cancer detection by topically spraying a gamma-glutamyltranspeptidase-activated fluorescent probe, Sci. Transl. Med. 110 (2011) 110r-119r.

[42] F. Wang, Y. Zhu, L. Zhou, L. Pan, Z. Cui, Q. Fei, S. Luo, D. Pan, Q. Huang, R. Wang, C. Zhao, H. Tian, C. Fan, Fluorescent in situ targeting probes for rapid imaging of ovarian-cancer-specific gamma-glutamyltranspeptidase, Angew. Chem. Int. Ed. Engl. 25 (2015) 7349-7353.

[43] S. Park, L. Soo-Yeon, Sang Mun Bae, Sang-Yeob Kim, Seung-Jae Myung, H. Kim, Indocyanine-based activatable fluorescence turn-on probe for $\gamma$-glutamyltranspeptidase and its application to the mouse model of colon cancer, ACS Sens. 1 (2016) 579-583.

[44] L. Li, W. Shi, Z. Wang, Q. Gong, H. Ma, Sensitive fluorescence probe with long analytical wavelengths for gamma-glutamyl transpeptidase detection in human serum and living cells, Anal. Chem. 16 (2015) 8353-8359.

[45] F. Liu, Z. Wang, W. Wang, J.G. Luo, L. Kong, Red-emitting fluorescent probe for detection of gamma-glutamyltranspeptidase and its application of real-time imaging under oxidative stress in cells and in vivo, Anal. Chem. 12 (2018) 7467-7473.

[46] P. Zhang, X.F. Jiang, X. Nie, Y. Huang, F. Zeng, X. Xia, S. Wu, A two-photon fluorescent sensor revealing drug-induced liver injury via tracking gamma-glutamyltranspeptidase (GGT) level in vivo, Biomaterials (2016) 46-56.

[47] Y. Liu, J. Tan, Y. Zhang, J. Zhuang, M. Ge, B. Shi, J. Li, G. Xu, S. Xu, C. Fan, C. Zhao, Visualizing glioma margins by real-time tracking of gamma-glutamyltranspeptidase activity, Biomaterials (2018) 1-10.

[48] B. Bai, C. Yan, Y. Zhang, Z. Guo, W. Zhu, Dual-channel near-infrared fluorescent probe for real-time tracking of endogenous c-glutamyl transpeptidase activity, Chem Comm 54 (2018) 12393-12396.

[49] Y.J. Reo, Y.W. Jun, S. Sarkar, M. Dai, K.H. Ahn, Ratiometric imaging of $\gamma$-glutamyl transpeptidase unperturbed by $\mathrm{pH}$, polarity, and viscosity changes: a benzocoumarin-based two photon fluorescent probe, Anal. Chem. 91 (2019) 14101-14108.

[50] R.J. Iwatate, M. Kamiya, K. Umezawa, H. Kashima, M. Nakadate, R. Kojima, Y. Urano, Silicon rhodamine-based near-infrared fluorescent probe for gammaglutamyltransferase, Bioconjug. Chem. 2 (2018) 241-244.

[51] L. Li, W. Shi, X. Wu, Q. Gong, X. Li, H. Ma, Monitoring gamma-glutamyl transpeptidase activity and evaluating its inhibitors by a water-soluble near-infrared fluorescent probe, Biosens. Bioelectron. (2016) 395-400.

[52] K. Gu, Y. Xu, H. Li, Z. Guo, S. Zhu, S. Zhu, P. Shi, T.D. James, H. Tian, W.H. Zhu, Real-time tracking and in vivo visualization of beta-galactosidase activity in colorectal tumor with a ratiometric near-infrared fluorescent probe, J. Am. Chem. Soc. 16 (2016) 5334-5340.

[53] L. Li, W. Shi, X. Wu, X. Li, H. Ma, In vivo tumor imaging by a gamma-glutamyl transpeptidase-activatable near-infrared fluorescent probe, Anal. Bioanal. Chem. 26 (2018) 6771-6777.

[54] Z. Luo, Z. Huang, K. Li, Y. Sun, J. Lin, D. Ye, H.Y. Chen, Targeted delivery of a $\gamma$-glutamyl transpeptidase activatable near-infrared-fluorescent probe for selective cancer imaging, Anal. Chem. 90 (2018) 2875-2883.

[55] V.L. Kinnula, C.L. Fattman, R.J. Tan, T.D. Oury, Oxidative stress in pulmonary fibrosis: a possible role for redox modulatory therapy, Am. J. Respir. Crit. Care Med. 4 (2005) 417-422.

[56] C. Xie, M.A. Lovell, S. Xiong, M.S. Kindy, J. Guo, J. Xie, V. Amaranth, T.J. Montine, W.R. Markesbery, Expression of glutathione-S-transferase isozyme in the SY5Y neuroblastoma cell line increases resistance to oxidative stress, Free Radic. Biol. Med. 1 (2001) 73-81.

[57] K.V. Pandit, D. Corcoran, H. Yousef, M. Yarlagadda, A. Tzouvelekis, K.F. Gibson, K. Konishi, S.A. Yousem, M. Singh, D. Handley, T. Richards, M. Selman, S.C. Watkins, A. Pardo, A. Ben-Yehudah, D. Bouros, O. Eickelberg, P. Ray, P.V. Benos, N. Kaminski, Inhibition and role of let-7d in idiopathic pulmonary fibrosis, Am. J. Respir. Crit. Care Med. 2 (2010) 220-229.

$\mathrm{Na} \mathrm{He}$ is currently a master candidate, joint-educated, at Binzhou Medical University and Yantai Institute of Coastal Zone Research, Chinese Academy of Sciences, since 2017. She received her BS from Binzhou Medical University, in 2017. Her current interest in research focuses on bioimaging.

Yude Wang is currently a master candidate, joint-educated, at Binzhou Medical University and Yantai Institute of Coastal Zone Research, Chinese Academy of Sciences, since 2018. His current interest in research focuses on fluorescence bioimaging.

Yan Huang is currently as a professor at School of Pharmacy, Binzhou Medical University. He received his Ph.D., joint-educated, at Shandong Normal University and Yantai Institute of Coastal Zone Research, Chinese Academy of Sciences, in 2019. His research interests focus on functional fluorescent probe, theranostics and functional nanomaterial.

Xiaoyan Wang is currently as an associate professor at School of Pharmacy, Binzhou Medical University. She received her Ph.D., joint-educated, at Shandong Normal University and Yantai Institute of Coastal Zone Research, Chinese Academy of Sciences, in 2017. Her research interests focus on the studies of functionalized nanoparticles for developing nanoscale biochemical analysis methods and molecular imprinting-based sample pretreatment technology.

Lingxin Chen has been a professor at Yantai Institute of Coastal Zone Research, Chinese Academy of Sciences, since 2009. He obtained his Ph.D. in analytical chemistry at Dalian Institute of Chemical Physics, Chinese Academy of Sciences, in 2003. During 2004-2009, he worked at Department of Chemistry, Tsinghua University, and Department of Applied Chemistry, Hanyang University, respectively. His research interests include the studies of novel properties of materials such as functionalized nanoparticles \& functional probe molecules for developing nanoscale biochemical analysis methods and molecular imprinting-based sample pretreatment technology.

Changjun Lv is now a vice president in Binzhou Medical University. He is a professor and serves in Affiliated Hospital of Binzhou Medical University as a chief physician. He received his MD from China Medical University in 1995. He is skilled in the diagnosis and treatment of respiratory diseases, especially in the diagnosis of interstitial lung diseases. $\mathrm{He}$ is an expert with China's State Council special allowance due to his excellent performance in clinical work. His research interests include diagnosis and therapy of idiopathic pulmonary fibrosis, drug developments and theranostics. 